\title{
Financial price fluctuations in a stock market model with many interacting agents
}

\author{
Ulrich Horst * \\ Institut für Mathematik \\ Humboldt-Universität zu Berlin \\ Unter den Linden 6 \\ D-10099 Berlin
}

13th June 2001

\begin{abstract}
We consider a financial market model with a large number of interacting agents. Investors are heterogeneous in their expectations about the future evolution of an asset price process. Their current expectation is based on the previous states of their "neighbors" and on a random signal about the "mood of the market". We analyze the asymptotics of both aggregate behaviour and asset prices. We give sufficient conditions for the distribution of equilibrium prices to converge to a unique equilibrium, and provide a microeconomic foundation for the use of diffusion models in the analysis of financial price fluctuations.
\end{abstract}

Key Words: behavioral finance, diffusion models, stochastic difference equations, interacting Markov chains

AMS 2000 subject classification: 60K35, 60J20, 60F05, 91B28

${ }^{*}$ The present paper is based on my Ph.D. thesis. I am grateful to my supervisor Hans Föllmer for numerous suggestions and for many motivating inputs. Thanks are due to Peter Bank, Dirk Becherer, Peter Leukert, Alexander Schied, and Ching-Tang Wu for innumerable and fruitful discussions. Financial support of Deutsche Forschungsgemeinschaft (SFB 373, "Quantification and Simulation of Economic Processes", Humboldt-Universität zu Berlin) is gratefully acknowledged. 


\section{Introduction}

In mathematical finance, the price process of a risky assert is usually modeled as the trajectory of a stochastic process on some underlying probability space $(\Omega, \mathcal{F}, \mathbb{P})$. Such a probabilistic approach was first initiated by Bachelier (1900), who introduced Brownian motion as a model for price fluctuations on the Paris stock exchange. As prices should stay positive, geometric Brownian motion is now widely used as the basic reference model in the analysis of financial price fluctuations. Kreps (1982) showed that such a diffusion model can be justified as the rational expectation equilibrium in a market with highly rational agents who all believe in this kind of price dynamics, and who instantaneously and rationally discount all available information into the present price. From this theoretical point of view large and sudden price fluctuations reflect rational changes in the valuation of an asset rather than irrational shifts in the sentiment of investors.

Traders, by contrast, often consider financial markets as being much less rational. Many practitioners believe that technical trading is possible, and that herd effects unrelated to economic fundamentals can cause bubbles or crashes. In particular, they are typically aware of the fact that asset prices may be driven by sudden shifts in the "mood of the market". In view of such market realities, it seems natural to regard price processes as the result of an interaction between many agents with bounded rationality. In particular, one should admit imitation and contagion effects in the formation of agents' expectations. When the set $\mathbb{A}$ of agents involved in the formation of stock prices becomes large, such an approach allows to bring in techniques from the theory of interacting Markov processes or from Markov random field theory. An early attempt into this direction has been made by Föllmer (1974) who considers a static model of endogenous preference formation.

In this paper we provide a unified probabilistic framework for modeling financial markets where the demand for a risky asset results from the interaction of a large number of traders. Following an approach suggested by Föllmer and Schweizer (1993), we are going to view the stock price process as a sequence of temporary price equilibria. We assume that the excess demand of agent $a \in \mathbb{A}$ in period $t$ depends on his current individual state $x_{t}^{a}$ reflecting, for example, his expectation about the future evolution of stock

prices. Such a distinction between different types of agents has been a major 
topic in the recent literature on financial markets and on dynamic microeconomic models; see, e.g. Brock and Hommes (1997), Kirman (1998), Lux (1998) or Lux and Marchesi (1999). For a given configuration of individual states $x=\left(x^{a}\right)_{a \in \mathbb{A}}$, the law of agent $a$ 's new state depends both on the current states $\left(x^{b}\right)_{b \in N(a)}$ of the agents in his social neighborhood $N(a)$ and on the average expectation in the whole population, i.e., on the "mood of the market". The mood of the market is described by the empirical distribution of individual agents' states or, more completely, by the empirical field $R(x)$ associated with the configuration $x$. We consider a situation where an individual agent has complete information about the actions chosen by agents in his reference group, but only has incomplete information about the average action throughout the entire population. Individual trader do not know $R(x)$ for sure, but only observe a random signal, e.g., a stock market index, whose distribution depends on $R(x)$.

In our financial market model, the random evolution of the mood of the market is the only component affecting the formation of temporary price equilibria. The microscopic process $\left\{x_{t}\right\}_{t \in \mathbb{N}}$ which describes the stochastic evolution of all the individual states generates via the macroscopic process $\left\{R\left(x_{t}\right)\right\}_{t \in \mathbb{N}}$ an endogenous random environment $\left\{\tilde{\varrho}_{t}\right\}_{t \in \mathbb{N}}$ for the evolution of the asset price process. The dynamics of the stock price process $\left\{p_{t}\right\}_{t \in \mathbb{N}}$ obeys a recursive relation of the form

$$
p_{t+1}=F\left(\tilde{\varrho}_{t+1}, p_{t}\right) \quad(t \in \mathbb{N}) .
$$

Our aim is to state conditions which ensure that the stock prices behave asymptotically in a stable manner. To this end, we shall first analyze the long run behaviour of the macroscopic process $\left\{R\left(x_{t}\right)\right\}_{t \in \mathbb{N}}$ which describes the dynamics of aggregate behaviour. Using results about the asymptotic behaviour of locally and globally interacting Markov chains provided in Horst (2000), we show that, in the limit of an infinite set $\mathbb{A}$ of traders, the dynamics on the level of aggregate behaviour can be described by a Markov chain associated with a suitable random system with complete connections. This allows us to state conditions on the behaviour of individual agents which guarantee that the mood of the market settles down in the long run. More precisely, we place a quantitative bound of the effects of social interaction in our economy, and show that the macroscopic process converges in law to a unique equilibrium distribution if the interaction between different agents is 
not too strong. In this case the sequence of equilibrium prices asymptotically evolves in a stationary random environment.

In the second part of this paper we study the asymptotics of the induced equilibrium price process. In a first step, we analyze an ergodic reference model, i.e., we assume that the driving sequence $\left\{\tilde{\varrho}_{t}\right\}_{t \in \mathbb{N}}$ is stationary and ergodic. Economically, this amounts to a situation where the mood is already in equilibrium. We show that the asset price process becomes asymptotically stationary if the destabilizing effects of the random environment on the dynamics of the price process is on average not too strong. Nonetheless, the price fluctuations in our financial market model may be highly volatile. This feature may be interpreted as the temporary occurrence of bubbles and crashes in a financial market model whose overall behaviour is ergodic.

From an economic point of view, however, such a stationarity assumption on the driving sequence might be rather restrictive. Instead, it seems more natural to investigate the asymptotic behaviour of asset prices under the assumption the at the mood is out of equilibrium, i.e., under the assumption that the random environment for the evolution of the price process is specified by a non-stationary stochastic process. However, given that the macroscopic process settles down in the long run, it is desirable to have sufficient conditions which ensure that asset prices are driven into a stationary regime. Assuming a simple log-linear structure for the excess demand functions leads to a class of log-linear price processes of the form

$$
\log p_{t+1}=f\left(\tilde{\varrho}_{t+1}\right) \log p_{t}+g\left(\tilde{\varrho}_{t+1}\right) \quad(t \in \mathbb{N}) .
$$

Under a suitable mean-contraction condition on the price dynamics $\left\{p_{t}\right\}_{t \in \mathbb{N}}$ specified by (2) we show that stock prices converge to a stationary regime if the mood of the market itself settles down in the long run. Armed with these results one could now try to analyze the structure of the equilibrium distribution, and to estimate, for example, the asymptotic variance of stock prices. Such an empirical analysis, however, is beyond the scope of this paper, and is left for future research.

In our final section we study a diffusion approximation of the discretetime price process $\left\{p_{t}\right\}_{t \in \mathbb{N}}$. Under simplifying assumption on excess demand functions of the agents, the sequence of temporary price equilibria can be approximated in law by a diffusion process $\left\{P_{t}\right\}_{t \geq 0}$ in continuous time. This result provides another microeconomic foundation for the use of 
diffusion processes in the analysis of financial price fluctuations. If the mood of the market is already in equilibrium, i.e., if the asset price process evolves in a stationary and ergodic random environment, then we find ourselves in the setting analyzed in Föllmer and Schweizer (1993), who obtained a continuous-time model from a sequence of suitably specified discrete-time processes evolving in an exogenously given random environment; see also Föllmer (1994). Proving a functional central limit theorem for stochastic processes evolving in a non-stationary random environment, we are able to extend the Föllmer-Schweizer model by (i) analyzing a situation were the driving sequence is derived endogenously, and (ii) by replacing the stationarity assumption on the mood of the market by an asymptotic stability condition. We show that the diffusion limit $\left\{P_{t}\right\}_{t \geq 0}$ converges to a stationary process whose invariant distribution can be given in closed form.

The rest of this paper is organized as follows. In Section 2 we introduce our financial market model. In Sections 3 and 4 we describe the dynamics of individual and aggregate behaviour, respectively. Section 5 analyzes the asymptotic behaviour of the discrete-time asset price process. In Section 6 we pass to a diffusion limit in continuous time. Section 7 concludes.

\section{The Microeconomic Model}

Let us describe a temporary equilibrium model for the price evolution of a speculative asset. We consider a financial market model with a countably infinite set $\mathbb{A}$ of economic agents trading a single risky asset. In reaction to a proposed stock price $p$ in period $t$, each agent $a \in \mathbb{A}$ forms an excess demand $z_{t}^{a}(p)$. Individual excess demand at time $t$ is to be thought of as the difference between individual demand and individual initial endowment in period $t$. The actual stock price $p_{t}$ at date $t$ will be determined by the equilibrium condition of zero total excess demand, and so the price process will be given by a sequence of temporary price equilibria $\left\{p_{t}\right\}_{t \in \mathbb{N}}$. We assume that the excess demand of an individual agent $a \in \mathbb{A}$ takes the form

$$
z_{t}^{a}(p)=z\left(p, p_{t}^{a}\right)
$$

Here, $p_{t}^{a}$ denotes an individual reference level of an agent $a$ at date $t$, e.g., his price expectation for the following period $t+1$. We shall assume that agents are heterogeneous in their individual reference levels. More precisely, 
at any given date $t \in \mathbb{N}$, the value $p_{t}^{a}$ depends on the present individual state $x_{t}^{a}$ of agent $a \in \mathbb{A}$, on the previous equilibrium price $p_{t-1}$, and on the proposed price $p$. Thus, it takes the form

$$
p_{t}^{a}(p)=g\left(x_{t}^{a}, p_{t-1}, p\right)
$$

for some measurable function $g: C \times \mathbb{R}^{2} \rightarrow \mathbb{R}$. Here

$$
C:=\left\{c_{1}, \ldots, c_{N}\right\}
$$

is a fixed set of individual states.

Example 2.1 ("Fundamentalists and Chartists") Let us consider a financial market model where the individual excess demand function takes the log-linear form

$$
z\left(p, p_{t}^{a}\right)=\log p_{t}^{a}-\log p
$$

as in Föllmer and Schweizer (1993). We put $C=\{-1,0,+1\}$ and consider a model with optimistic $\left(x_{t}^{a}=+1\right)$ and pessimistic $\left(x_{t}^{a}=-1\right)$ information traders ("fundamentalists") and with chartists $\left(x_{t}^{a}=0\right)$.

We assume that a fundamentalist's subjective perception $\log L+x_{t}^{a}$ of the current value of the asset fluctuates around some long run fundamental value $\log L$. Independent of the proposed price, his expectation is based on the idea that the next price will move closer to his actual benchmark for the fair value of the stock. More precisely, his reference level takes the form

$$
\log p_{t}^{a}=\log p_{t-1}+c_{f}\left(\log L+x_{t}^{a}-\log p_{t-1}\right) \quad\left(c_{f}>0\right) .
$$

The chartist, on the other hand, takes the proposed price as a serious signal about the future evolution of the security price and replaces $L$ in (6) by $p$. Thus, his price expectation takes the form

$$
\log p_{t}^{a}=\log p_{t-1}+c_{c}\left(\log p-\log p_{t-1}\right) \quad\left(c_{c}>0\right) .
$$

In equilibrium, i.e., for $p=p_{t}$, a chartist forecasts the future evolution of the asset price process from past observations.

In our model, the dynamics of the price process will be induced by an underlying microscopic process $\left\{x_{t}\right\}_{t \in \mathbb{N}}=\left\{\left(x_{t}^{a}\right)_{a \in \mathbb{A}}\right\}_{t \in \mathbb{N}}$ which describes the 
stochastic evolution of all the individual states. The process $\left\{x_{t}\right\}_{t \in \mathbb{N}}$ takes values in the configuration space

$$
E:=C^{\mathbb{A}}=\left\{x=\left(x^{a}\right)_{a \in \mathbb{A}}: x^{a} \in C\right\} .
$$

We shall view $E$ as a compact metric space which is equipped with the product topology and denote by $\mathcal{E}$ the product- $\sigma$-field on $E$.

Let us now concentrate on the resulting stock price process $\left\{p_{t}\right\}_{t \in \mathbb{N}}$. In a first step, we consider a situation where only finitely many investors are active on the market. To this end, we fix a sequence $\left\{\mathbb{A}_{n}\right\}_{n \in \mathbb{N}}$ of finite subsets of $\mathbb{A}$ satisfying $\mathbb{A}_{n} \uparrow \mathbb{A}$ as $n \uparrow \infty$. If only the traders in $\mathbb{A}_{n}$ are active on the market, the equilibrium stock price $p_{t}^{n}$ at date $t$ is determined by the market clearing condition of zero total excess demand, i.e., $p_{t}^{n}$ is given by an implicit solution of the equation

$$
\sum_{a \in \mathbb{A}_{n}} z_{t}^{a}\left(p_{t}^{n}\right)=0
$$

In view of (3) and (4), and in terms of per capita excess demand, we can rewrite (8) as

$$
\int_{C} z\left(p_{t}^{n}, g\left(c, p_{t-1}^{n}, p_{t}^{n}\right)\right) \varrho^{n}\left(x_{t}\right)(d c)=0
$$

Here,

$$
\varrho^{n}\left(x_{t}\right):=\frac{1}{\left|\mathbb{A}_{n}\right|} \sum_{a \in \mathbb{A}_{n}} \delta_{x_{t}^{a}}(\cdot) \in \mathcal{M}(C)
$$

denotes the empirical distribution of the states assumed by the traders $a \in$ $\mathbb{A}_{n}$ in period $t$, and $\mathcal{M}(C)$ is the class of all probability measures on $C$.

For any $n \in \mathbb{N}$, the implicit equation (9) defines the sequence of temporary equilibrium prices $\left\{p_{t}^{n}\right\}_{t \in \mathbb{N}}$ in the case of a finite set $\mathbb{A}_{n}$ of traders who are involved in the formation of equilibrium prices. In order to extend the construction of equilibrium prices to the infinite system of all agents, we are going to restrict our state space $E$ to the class $E_{0}$ of configurations $x=\left(x^{a}\right)_{a \in \mathbb{A}} \in E$ which admit the weak limit

$$
\varrho(x):=\lim _{n \rightarrow \infty} \varrho^{n}(x) .
$$


That is,

$$
E_{0}:=\left\{x \in E: \exists \lim _{n \rightarrow \infty} \frac{1}{\left|A_{n}\right|} \sum_{a \in \mathbb{A}_{n}} \delta_{x^{a}}(\cdot) \in \mathcal{M}(C)\right\}
$$

Thus, for $x \in E_{0}$ the empirical distribution $\varrho(x)$ associated with the configuration $x$ exists. We will call $\varrho\left(x_{t}\right)$ the mood of the market at time $t$.

Even though we consider an economy with a countably infinite set of agents, it seems reasonable to view the set of agents who are directly involved in the formation of the equilibrium price at date $t$ as a random subset $A_{t} \subset \mathbb{A}$ of representative agents. By saying that $A_{t}$ is a set of representative agents we mean that the empirical distribution $\tilde{\varrho}_{t}$ of the states assumed by the traders in $A_{t}$ is a random variable whose conditional law

$$
\tilde{Q}\left(\varrho\left(x_{t}\right) ; \cdot\right)
$$

is described by a stochastic kernel $\tilde{Q}$ from $\mathcal{M}(C)$ to $\mathcal{M}(C)$.

We are now ready to define the equilibrium price $p_{t}$ at date $t$. In the limit of an infinite set of agents, $p_{t}$ will be given as an implicit solution of the equation

$$
\int_{C} z\left(p_{t}, g\left(c, p_{t-1}, p_{t}\right)\right) \tilde{\varrho}_{t}(d c)=0
$$

which may be viewed as the limiting form of (9). In order to obtain a uniquely defined stock price process, we impose the following condition on the excess demand function $z$ :

Assumption 2.2 There exists a measurable function $F: \mathcal{M}(C) \times \mathbb{R} \rightarrow \mathbb{R}$ such that the implicit equation

$$
\int_{C} z\left(p^{*}, g\left(c, p, p^{*}\right)\right) \tilde{\varrho}(d c)=0
$$

admits a unique solution $p^{*}=F(\tilde{\varrho}, p)$ for any pair $(\tilde{\varrho}, p) \in \mathcal{M}(C) \times \mathbb{R}$.

Example 2.3 Let us return to the log-linear demand structure described by (5) together with (6) and (7). For a given configuration $x_{t} \in E_{0}$, the empirical distribution $\tilde{\varrho}_{t}$ is described by the proportions $\tilde{\varrho}_{t}^{+}, \tilde{\varrho}_{t}^{-}$and $\tilde{\varrho}_{t}^{0}$ of optimistic and pessimistic fundamentalists and of chartists, respectively. In 
the limit of an infinite set of agents the market clearing condition (11) implies the following linear structure for the evolution of the logarithmic stock price process:

$$
\log p_{t+1}=f\left(\tilde{\varrho}_{t+1}\right) \log p_{t}+g\left(\tilde{\varrho}_{t+1}\right),
$$

where

$$
f\left(\tilde{\varrho}_{t}\right):=\frac{1-c_{f}\left(\tilde{\varrho}_{t}^{+}-\tilde{\varrho}_{t}^{-}\right)-c_{c} \tilde{\varrho}_{t}^{0}}{1-c_{c} \tilde{\varrho}_{t}^{0}}, \quad g\left(\varrho\left(x_{t}\right)\right):=\frac{c_{f}\left(\log L+\tilde{\varrho}_{t}^{+}-\tilde{\varrho}_{t}^{-}\right)}{1-c_{c} \tilde{\varrho}_{t}^{0}} .
$$

In Section 5.2 we will state conditions which guarantee that the sequence $\left\{\log p_{t}\right\}_{t \in \mathbb{N}}$ converges to a stationary regime if the mood of the market settles down as $t \rightarrow \infty$. Observe, however, that for $c_{c}>1$, the mappings $f$ and $g$ have a singularity. If the fraction of chartists who are actually involved in the formation of price equilibria comes close to the critical value $\tilde{\varrho}=\frac{1}{c_{c}}$, then the price process becomes highly volatile. A slight change in the current proportion of chartists may have a lasting effect on asset prices.

Given that our Assumption 2.2 holds true, the stock price process $\left\{p_{t}\right\}_{t \in \mathbb{N}}$ is defined by the recursive relation

$$
p_{t+1}=F\left(\tilde{\varrho}_{t+1}, p_{t}\right) \quad(t \in \mathbb{N}) .
$$

In our setting, the evolution of the empirical distribution of agents' states is therefore the only component affecting the formation of temporary price equilibria. The process $\left\{x_{t}\right\}_{t \in \mathbb{N}}$ generates - via the aggregate quantities $\left\{\varrho\left(x_{t}\right)\right\}$ and $\left\{\tilde{\varrho}_{t}\right\}_{t \in \mathbb{N}}-$ an endogenous random environment for the evolution of the stock price process. Our goal is to analyze the long run behaviour of the asset price process $\left\{p_{t}\right\}_{t \in \mathbb{N}}$. To this end, we have to analyze the asymptotics of the driving sequence $\left\{\tilde{\varrho}_{t}\right\}_{t \in \mathbb{N}}$. In the next section, we begin by describing the dynamics of the microscopic process $\left\{x_{t}\right\}_{t \in \mathbb{N}}$. In Section 4 we state conditions on the behaviour of individual agents which guarantee that the mood of the market settles down in the long run. In this case the price process asymptotically evolves in a stationary random environment. In Section 5 we show that this yields weak convergence of the asset price process if the excess demand functions take the log-linear form (5). 


\section{The Dynamics of Individual Behaviour}

Let us now specify the dynamics of the microscopic process $\left\{x_{t}\right\}_{t \in \mathbb{N}}$. We assume that, in each period $t$, any agent $a \in \mathbb{A}$ chooses his next state $x_{t+1}^{a}$ at random according to some probability law which depends on the present configuration of individuals states $x_{t}=\left(x_{t}^{a}\right)_{a \in \mathbb{A}}$. More precisely, the process $\left\{x_{t}\right\}_{t \in \mathbb{N}}$ will be described by a Markov chain,

$$
\Pi(x ; d y)=\prod_{a \in \mathbb{A}} \pi^{a}\left(x ; d y^{a}\right),
$$

on a suitable subset of the configuration space $E=C^{\mathbb{A}}$.

The dependence of the probability law $\pi^{a}$ on the present configuration can have both a local and a global component. Local dependence in the choice of an individual agent $a \in \mathbb{A}$ refers to dependence of $\pi^{a}$ on some set of neighbors $b \in N(a)$. In particular, introducing the notion of local interaction requires to endow the countable set $\mathbb{A}$ with the structure of a graph, where the agents are the nodes and where interactive links between certain pairs of agents exist. Since we shall also admit a global component in the interaction, we will need to consider ergodic averages over the whole graph of agents. Therefore, we limit ourselves to the case best understood so far, where $\mathbb{A}$ carries a lattice structure, and assume that the agents are located on the d-dimensional integer lattice, i.e., $\mathbb{A}:=\mathbb{Z}^{d}$.

The neighborhood or reference group $N(a)$ associated with agent $a \in \mathbb{A}$ is given by the set

$$
N(a):=\{b \in \mathbb{A}:|a-b| \leq l<\infty\} .
$$

Here, $|\cdot|$ denotes the Euclidean distance on $\mathbb{R}^{d}$ and $l \in \mathbb{N}$ is a fixed "interaction radius". In terms of such peer groups we can model situations where the current reference level $p_{t+1}^{a}$ of agent $a$, e.g., his expectation about the future evolution of the stock price, is influenced by the previous states $\left(x_{t}^{b}\right)_{b \in N(a)}$ of his neighbors. Note that in our model any agent affects the next state of just $2^{d l}$ other persons, and so no individual trader is able to influence the mood of the whole market in one single period. In this sense, we consider a financial market model with many small investors.

In real financial markets, the behaviour of an individual trader is, of course, not only influenced by the actions chosen by the agents in his reference group, but also depends on the current mood of the market, i.e., on the 
average behaviour throughout the entire population. However, investors do typically not have complete information about the empirical distribution of individual agents' states in a given period. Instead, it seems reasonable to assume that they only have incomplete information about $\varrho\left(x_{t}\right)$ in the sense that they receive a common noisy signal $s_{t}$ about the average expectation at date $t$. For example, $s_{t}$ could be a signal about the fraction of chartists or optimistic information traders who are active on the market in period $t$ and may be revealed by, e.g., a stock market index. Thus, it makes immediate sense to introduce an additional dependence on signals about "global properties" of the current configuration, e.g., a dependence on signals about the mood of the market, into the interaction. The following simple voter model illustrates this approach.

Example 3.1 Let us put $C=\{0,1\}$. We assume that an inidividual investor reacts both to the currents states of his neighbors and to a random signal $s \in[0,1]$ about the average action throughout the entire population. For any fixed signal $s$, an agent chooses his next state according to a transition law $\pi_{s}^{a}$ from $E_{0}$ to $C$ which is described by the convex combination

$$
\pi_{s}^{a}(x ; 1)=\alpha p\left(x^{a}\right)+\beta m^{a}(x)+\gamma s .
$$

Here, $\alpha p\left(x^{a}\right)$ measures the dependence of agent a's new state on his current one, and $m^{a}(x)$ is the proportion of ' 1 ' in the neighborhood $N(a)$. Moreover, $s \in[0,1]$ denotes a commonly known random signal about the empirical average

$$
m(x):=\lim _{n \rightarrow \infty} \frac{1}{\left|\mathbb{A}_{n}\right|} \sum_{a \in \mathbb{A}_{n}} x^{a}
$$

associated with the configuration $x \in E_{0}$. The conditional law $Q\left(m\left(x_{t}\right) ; \cdot\right)$ of the signal $s_{t}$ given the average $m\left(x_{t}\right)$ is described by a signal kernel $Q$ on $[0,1]$. Due to the linear structure of the transition probabilities $\pi_{s}^{a}$, the law of large numbers shows that, for any given signal sequence $\left\{s_{t}\right\}_{t \in \mathbb{N}}$, the process $\left\{m\left(x_{t}\right)\right\}_{t \in \mathbb{N}}$ satisfies almost surely the deterministic dynamics

$m\left(x_{t+1}\right)=u\left(m\left(x_{t}\right), s_{t}\right):=\alpha\left\{m\left(x_{t}\right) p(1)+\left(1-m\left(x_{t}\right)\right) p(0)\right\}+\beta m\left(x_{t}\right)+\gamma s_{t}$.

In this model, the sequence of empirical averages $\left\{m\left(x_{t}\right)\right\}_{t \in \mathbb{N}}$ may therefore be viewed as a Markov chain on the state space $[0,1]$. In Sections 4 and 5, 
respectively, we provide conditions which ensure that both the Markov chain $\left\{m\left(x_{t}\right)\right\}_{t \in \mathbb{N}}$ and the induced price process $\left\{p_{t}\right\}_{t \in \mathbb{N}}$ converge in law to a unique equilibrium.

The next example shows that the dynamics of the sequence $\left\{m\left(x_{t}\right)\right\}_{t \in \mathbb{N}}$ typically cannot be described by a Markov chain.

Example 3.2 Consider the following generalization of the voter model (15). For $x \in E_{0}$ and $s \in[0,1]$, the individual transition probabilities are described by a measurable mapping $g_{s}: C^{|N(a)|} \rightarrow[0,1]$ in the sense that

$$
\pi_{s}^{a}(x ; 1)=g_{s}\left(\left\{x^{b}\right\}_{b \in N(a)}\right) .
$$

Typically, we cannot expect that there exist a function $u:[0,1] \times[0,1] \rightarrow[0,1]$ such that $m\left(x_{t+1}\right)=u\left(m\left(x_{t}\right), s_{t}\right)$. Nevertheless, we will show that the mood of the market settles down in the long run if the dependence of the mapping $g_{s}$ on $x^{b}(b \in N(a))$ is not too strong; cf. Example 4.6.

Due to the local dependence of the individual transition laws $\pi^{a}$ on the current configuration, the dynamics of the mood of the market in general cannot be described by a Markov chain. In order to analyze the long run behaviour of aggregate behaviour in our financial market model, we need a more general mathematical framework which we are now going to specify. To start with, we introduce the family of shift-transformations $\theta_{a}(a \in \mathbb{A})$ on $E$ defined by $\left(\theta_{a} x\right)(b)=x^{a+b}$.

Definition 3.3 (i) We denote by $\mathcal{M}(E)$ the class of all probability measures on $E$. A probability measure $\mu \in \mathcal{M}(E)$ will also be called a random field.

(ii) A random field $\mu \in \mathcal{M}(E)$ is called homogeneous, if $\mu$ is invariant under the shift maps $\left(\theta_{a}\right)_{a \in \mathbb{A}}$. By

$$
\mathcal{M}_{h}(E):=\left\{\mu \in \mathcal{M}(E): \mu=\mu \circ \theta_{a} \text { for all } a \in \mathbb{A}\right\}
$$

we denote the class of all homogeneous random fields $\mu$ on $E$.

(iii) A homogeneous random field $\mu \in \mathcal{M}_{h}(E)$ is called ergodic, if $\mu$ satisfies a 0-1-law on the $\sigma$-field of all shift invariant events. The class of all ergodic probability measures $\mu$ on $E$ is denoted by $\mathcal{M}_{e}(E)$. 
For a given $n \in \mathbb{N}$ we put $\mathbb{A}_{n}:=[-n, n]^{d} \cap \mathbb{A}$, and denote by $E_{e}$ the set of all configuration $x \in E$ such that the empirical field $R(x)$, defined as the weak limit

$$
R(x):=\lim _{n \rightarrow \infty} \frac{1}{\left|\mathbb{A}_{n}\right|} \sum_{a \in \mathbb{A}_{n}} \delta_{\theta_{a} x}(\cdot),
$$

exists and belongs to $\mathcal{M}_{e}(E)$. The empirical field $R(x)$ carries all macroscopic information carried in the configuration of individual states $x=$ $\left(x^{a}\right)_{a \in \mathbb{A}} \in E_{e}$. In particular, the empirical distribution

$$
\varrho(x)=\lim _{n \rightarrow \infty} \frac{1}{\left|\mathbb{A}_{n}\right|} \sum_{a \in \mathbb{A}_{n}} \delta_{x^{a}}(\cdot),
$$

i.e., the mood of the market associated with the configuration $x \in E_{e}$, is given as the one-dimensional marginal distribution of $R(x)$.

Consider the product kernel $\Pi_{s}$ on $E$ governed by the transition laws $\pi_{s}^{a}$ in (16). Proposition 4.1 below shows that the measure $\Pi_{s}(x ; \cdot)\left(x \in E_{e}\right)$ is concentrated on the set $E_{e}$ and that the empirical average satisfies

$$
m(y)=u(R(x), s):=\int \pi_{s}(x ; 1) R(x)(d z) \quad \Pi_{s}(x ; \cdot) \text {-a.s. }
$$

Thus, we have to consider the full dynamics of the sequence of empirical fields $\left\{R\left(x_{t}\right)\right\}_{t \in \mathbb{N}}$ even if, as in Example 3.2, the behaviour of agent $a \in \mathbb{A}$ depends on $R(x)$ only on the empirical average $m(x)$. Theorem 4.2 below shows that, in contrast to the sequence of empirical distributions $\left\{\varrho\left(x_{t}\right)\right\}_{t \in \mathbb{N}}$, the dynamics of the sequence of empirical field $\left\{R\left(x_{t}\right)\right\}_{t \in \mathbb{N}}$ can indeed be described by a Markov chain. Our aim is to formulate conditions on the transition laws $\pi^{a}$, i.e., on the behaviour of individual agents, which guarantee that the sequence of empirical fields $\left\{R\left(x_{t}\right)\right\}_{t \in \mathbb{N}}$ converges in law to a unique equilibrium distribution. In case we will say that the mood of the market settles down in the long run.

To this end, we shall now be more specific about the structure of the transition probabilities $\pi^{a}$. We assume that the interactive influence of the present configuration $x \in E_{e}$ on agent $a$ is felt both through the local situation $\left(x^{b}\right)_{b \in N(a)}$ in his neighborhood, and through a random signal about the average situation throughout the entire population $\mathbb{A}$. The average situation is described by the empirical field $R(x)$ associated with $x \in E_{e}$. The 
conditional law

$$
Q(R(x) ; \cdot)
$$

of the signal $s$ given the empirical field $R(x)$ is specified by a stochastic kernel $Q$ from $\mathcal{M}_{h}(E)$ to $S$, where $S$ is a finite signal space. ${ }^{1}$ The kernel $Q$ will be called the signal kernel.

We also assume that interaction between different agents is spatially homogeneous. This means that all traders react in the same manner both to the actions previously chosen by the agents in their reference group and to the signal about aggregate behaviour. Thus, for a fixed signal $s \in S$ and configuration $x \in E$, the probability that agent $a \in \mathbb{A}$ switches to state $c \in C$ in the following period is given by

$$
\pi_{s}^{a}(x ; c)=\pi_{s}\left(\theta_{a} x ; c\right)
$$

where $\pi_{s}(x ; \cdot)$ is a stochastic kernel from $E \times S$ to $C$.

Assumption 3.4 The probability laws $\left\{\pi_{s}(x ; \cdot)\right\}_{x \in E}$ satisfy a spatial Markov property of order $l$ in their dependence on the present configuration:

$$
\pi_{s}\left(\theta_{a} x ; \cdot\right)=\pi_{s}\left(\theta_{a} y ; \cdot\right) \quad \text { if } \theta_{a} x=\theta_{a} y \text { on } N(a) .
$$

Economically, this condition means that, for any fixed signal about the mood of the market, the new state of an agent only depends on the previous states of this neighbors.

Let us now fix a signal $s \in S$ and a configuration $x \in E$. It follows from our Assumption 3.4 and from (19) that

$$
\Pi_{s}(x ; \cdot):=\prod_{a \in \mathbb{A}} \pi_{s}\left(\theta_{a} x ; \cdot\right)
$$

defines a Feller kernel on the configuration space $E$ which is spatially homogeneous:

$$
\int_{E} f(y) \Pi_{s}\left(\theta_{a} x ; d y\right)=\int_{E} f\left(\theta_{a} y\right) \Pi_{s}(x ; d y)
$$

\footnotetext{
${ }^{1}$ The assumption that $S$ is finite merely simplifies notation. Our analysis also goes through under the assumption that $(S, \mathcal{S})$ is an arbitrary measurable space.
} 
for all $a \in \mathbb{A}$ and $f \in \mathcal{C}(E)$. In particular, the individual transition laws $\pi_{s}$ together with the signal kernel $Q$ determine a stochastic kernel

$$
\Pi(x ; \cdot):=\int_{S} \Pi_{s}(x ; \cdot) Q(R(x) ; d s)
$$

from $E_{e}$ to $E$. In fact, Proposition 4.1 below shows that $\Pi$ may be viewed as a stochastic kernel on the configuration space $E_{e}$. In contrast to $\Pi_{s}$, however, the kernel $\Pi$ typically does not have the Feller property, due to the macroscopic dependence on the present configuration $x$ via the empirical field $R(x)$. Thus, we cannot apply the method in Vasserstein (1969) in order to study the long run behaviour of the processes $\left\{x_{t}\right\}_{t \in \mathbb{N}}$ and $\left\{R\left(x_{t}\right)\right\}_{t \in \mathbb{N}}$. Instead, we will use results about the asymptotic behaviour of locally and globally interacting Markov processes recently reported in Föllmer and Horst (2001) and Horst (2001a).

\section{The Dynamics of Aggregate Behaviour}

In this section we are going to formulate conditions on the individual transition laws $\pi_{s}$ and on the signal kernel $Q$ which guarantee that the mood of the market settles down in the long run. ${ }^{2}$ In a first step, we use a law of large numbers for the random fields $\Pi_{s}$ in order to view $\Pi$ as a stochastic kernel on the configuration space $E_{e}$. For the proof we refer to Horst (2001a), Proposition 3.1.

Proposition 4.1 For all configurations $x \in E_{e}$, and for any signal $s \in S$, we have $\Pi_{s}\left(x ; E_{e}\right)=1$. For $\Pi_{s}(x ; \cdot)$-a.e. $y \in E_{e}$, the empirical field $R(y)$ takes the form

$$
R(y)=u(R(x), s):=\int_{E} \Pi_{s}(y ; \cdot) R(x)(d y) .
$$

In view of the this Proposition, we use $E_{e}$ as the state space for our microscopic process $\left\{x_{t}\right\}_{t \in \mathbb{N}}$. We denote by $\mathbb{P}_{x}$ the distribution of the Markov chain $\left\{x_{t}\right\}_{t \in \mathbb{N}}$ with initial state $x \in E_{e}$. Since a configuration $x \in E_{e}$ induces

\footnotetext{
${ }^{2}$ The asymptotics of the Markov chain $\Pi$ is explicitly analyzed in Horst (2001a). In order to keep the present paper self-contained, we summarize some of the results, but omit the proofs.
} 
an ergodic empirical field $R(x)$, the microscopic process $\left\{x_{t}\right\}_{t \in \mathbb{N}}$ induces $\mathbb{P}_{x^{-}}$ a.s. the macroscopic process $\left\{R\left(x_{t}\right)\right\}_{t \in \mathbb{N}}$ with state space $\mathcal{M}_{e}(E)$. The law of the random variable $R\left(x_{t+1}\right)$ depends on the microscopic configuration $x_{t}$ in period $t$ only through the empirical field $R\left(x_{t}\right)$. Thus, it is easily seen that the following result holds true.

Theorem 4.2 (i) Under the measure $\mathbb{P}_{x}$ the macroscopic process is a Markov chain on the state space $\mathcal{M}_{e}(S)$ with initial value $R(x)$.

(ii) For any given initial configuration $x \in E_{e}$, and for each fixed signal sequence $\left\{s_{t}\right\}_{t \in \mathbb{N}}$ our macroscopic process satisfies

$$
R\left(x_{t+1}\right)=u\left(\cdot, s_{t}\right) \circ \cdots \circ u\left(\cdot, s_{1}\right) \circ u\left(R(x), s_{0}\right) \quad \mathbb{P}_{x}-a . s .
$$

for any $t \in \mathbb{N}$.

Since, conditioned on the environment $\left\{s_{t}\right\}_{t \in \mathbb{N}}$, the macroscopic process follows almost surely a deterministic dynamics, we propose a "random system with complete connections" (henceforth RSCC) as a suitable mathematical framework for analyzing the dynamics of aggregate behaviour in our financial market model. Let us recall the notion of a RSCC.

Definition 4.3 Let $\left(M_{1}, d_{M_{1}}\right)$ be a metric space and $\left(M_{2}, \mathcal{M}_{2}\right)$ be a measurable space. Let $Z$ denote a stochastic kernel from $M_{1}$ to $\mathcal{M}_{2}$, and let $v: M_{1} \times M_{2} \rightarrow M_{1}$ be a measurable mapping. Following Iosefescu and Theodorescu (1968), we call the quadruple

$$
\Sigma:=\left(\left(M_{1}, d_{M_{1}}\right),\left(M_{2}, \mathcal{M}_{2}\right), Z, v\right)
$$

a random system with complete connections. ${ }^{3}$

(i) Given an initial value $\xi \in M_{1}$, a RSCC induces two stochastic processes $\left\{\xi_{t}\right\}_{t \in \mathbb{N}}$ and $\left\{\zeta_{t}\right\}_{t \in \mathbb{N}}$ on the canonical probability space $\left(\hat{\Omega}, \hat{\mathcal{F}}, \hat{\mathbb{P}}_{\xi}\right)$ taking values in $M_{1}$ and in $M_{2}$, respectively, by

$$
\xi_{t+1}=v\left(\xi_{t}, \zeta_{t}\right) \quad \text { and } \quad \hat{\mathbb{P}}_{\xi}\left(\zeta_{t} \in \cdot \mid \xi_{t}, \zeta_{t-1}, \xi_{t-1}, \zeta_{t-2}, \ldots\right)=Z\left(\xi_{t} ; \cdot\right)
$$

\footnotetext{
${ }^{3}$ We refer the reader to the books of Iosefescu and Theodorescu (1968) and Norman (1972) for a detailed discussion of RSCCs. Under the different name "iterated function systems", this class of processes is also studied in, e.g., Barnsley, Demko, Elton, and Geronimo (1988) and Lasota and York (1994).
} 
Here, $\xi_{0}=\xi \hat{\mathbb{P}}_{\xi^{-}}$a.s. These processes are called the associated Markov process and the signal sequence, respectively.

(ii) We say that a random system with complete connections is a distancediminishing model, if the transformation $v: M_{1} \times M_{2} \rightarrow M_{1}$ satisfies the contraction condition

$$
d_{M_{1}}(v(\xi, \zeta), v(\hat{\xi}, \zeta)) \leq \theta d_{M_{1}}(\xi, \hat{\xi})
$$

for some constant $\theta<1$.

Let $\left\{\xi_{t}\right\}_{t \in \mathbb{N}}$ be a Markov chain associated with a random system with complete connections $\Sigma=\left(\left(M_{1}, d_{M_{1}}\right),\left(M_{2}, \mathcal{M}_{2}\right), Z, v\right)$. For any fixed signal sequence $\left\{\zeta_{t}\right\}_{t \in \mathbb{N}}$, we have that

$$
\xi_{t+1}=v\left(\cdot, \zeta_{t}\right) \circ \cdots \circ v\left(\cdot, \zeta_{1}\right) \circ u\left(\xi, \zeta_{0}\right) \quad \hat{\mathbb{P}}_{\xi^{-}} \text {a.s. }
$$

Let us now fix an initial configuration $x \in E_{e}$. In view of (20) and (23), our macroscopic process may be viewed as the Markov chain with starting point $R(x)$ associated with the random system with complete connections

$$
\Sigma^{*}:=\left(\left(\mathcal{M}_{h}(E), d\right),(S, \mathcal{S}), Q, u\right),
$$

where the mapping $u: \mathcal{M}_{h}(E) \times S \rightarrow \mathcal{M}_{h}(E)$ is defined by

$$
u(R, s):=\int_{E} \Pi_{s}(x ; \cdot) R(d x),
$$

and where $d$ denotes the metric introduced in Föllmer and Horst (2001) which induces the weak topology on $\mathcal{M}_{h}(E)$; see also (2.53) in Horst (2000). ${ }^{4}$ Indeed, let us denote by $\left(\left\{\xi_{t}\right\}_{t \in \mathbb{N}},\left(\hat{\mathbb{P}}_{\varrho}\right)_{\varrho \in \mathcal{M}_{h}(E)}\right)$ the Markov chain on $\mathcal{M}_{h}(E)^{\mathbb{N}}$ associated with $\Sigma^{*}$. An easy induction argument shows that

$$
\mathbb{P}_{x}\left[\left\{R\left(x_{t}\right)\right\}_{t \in \mathbb{N}} \in B\right]=\hat{\mathbb{P}}_{R(x)}\left[\left\{\xi_{t}\right\}_{t \in \mathbb{N}}, \in B\right]
$$

for all $B$ in $\mathcal{B}^{\mathbb{N}}$, the Borel- $\sigma$-field on $\mathcal{M}_{h}(E)^{\mathbb{N}}$. In order to guarantee asymptotic stability of our macroscopic process, it is therefore enough to state

\footnotetext{
${ }^{4}$ Observe that we are going to view the macroscopic process as a Markov chain on the compact metric space $\left(\mathcal{M}_{h}(E), d\right)$ even though $R\left(x_{t}\right) \in \mathcal{M}_{e}(E)$ for all $t \in \mathbb{N}$. We refer to Horst (2001a) for a detailed discussion of this issue.
} 
conditions which ensure that the Markov chain $\left\{\xi_{t}\right\}_{\in \mathbb{N}}$ converges in law to a unique equilibrium. Due to Theorem 4.1.2 in Norman (1972), the process $\left\{\xi_{t}\right\}_{t \in \mathbb{N}}$ converges in law whenever the random system $\Sigma^{*}$ is distance diminishing in the sense of Definition 4.3 (ii), and given that the signal kernel $Q$ satisfies the following conditions.

Assumption 4.4 (i) The signal kernel $Q$ from $\mathcal{M}_{h}(E)$ to $S$ satisfies a uniform Lipschitz condition in the sense that

$$
\sup _{s \in S, \xi \neq \hat{\xi}} \frac{\mid Q(\xi ; s)-Q(\hat{\xi} ; s)}{d(\xi, \hat{\xi})} \leq L<\infty .
$$

(ii) We have that $\inf _{\xi, i} Q\left(\xi, s_{i}\right)>0$.

Remark 4.5 Economically, our Assumption 4.4 (ii) states that the agents receive every signal with small but positive probability, regardless of the prevailing mood of the market. Both parts (i) and (ii) of the above assumption exclude situations where the traders have complete information about the aggregate behaviour throughout the entire population.

Let us now state a condition on the individual transition laws $\pi_{s}$ which guarantees that the random system $\Sigma^{*}$ is distance diminishing. To this end, we define, for any pair $(a, s) \in \mathbb{A} \times S$, a vector $r_{a}^{s}=\left(r_{a, b}^{s}\right)_{b \in \mathbb{A}}$ with components

$$
r_{a, b}^{s}=\sup \left\{\frac{1}{2}\left\|\pi_{s}(x ; \cdot)-\pi_{s}(y ; \cdot)\right\|: x=y \text { off } a-b\right\} \quad(b \in \mathbb{A}),
$$

where $\left\|\pi_{s}(x ; \cdot)-\pi_{s}(y ; \cdot)\right\|$ denotes the total variation distance of the signed measure $\pi_{s}(x ; \cdot)-\pi_{s}(y ; \cdot)$ on the set $C$. Since we assume that the interaction between different agents is spatially homogeneous, it is easily seen that

$$
r_{a, b}^{s}=r_{a-b, 0}^{s} \quad(a, b \in \mathbb{A}) .
$$

Economically, the quantity $r_{a, 0}^{s}$ measures, for a fixed signal $s \in S$, the dependence of the new state of agent 0 on the current state of agent $a$. In order to ensure convergence of the mood of the market, we place a quantitative 
bound on the effect of social interactions in our economy. We shall say that Weak Social Interaction prevails if

$$
\alpha:=\sup _{s} \sum_{a} r_{a, 0}^{s}<1
$$

i.e., if the dependence of an agent's action on the current configuration is not too strong. Our weak social interaction assumption excludes situations where an agents imitates with probability one the behaviour of any of his neighbors.

Before we prove the main result of this section, we consider a simple example, where our weak social interaction assumption can indeed we verified.

Example 4.6 Let us fix a configuration $x \in E_{e}$ and a signal $s \in S$, and assume that the probability that an agent switches to state ' 1 ' is given by (16). Suppose that the mapping $g_{s}: C^{|N(a)|} \rightarrow[0,1]$ is differentiable, and denote the partial derivative with respect to $x^{b}$ by $g_{s}^{b}$. If

$$
\max _{s} \sum_{a \in N(0)} \max \left\{\left|g_{s}^{a}\left(\left(x^{b}\right)_{b \in N(0)}\right)\right|: x^{b} \in C\right\}<1,
$$

then our weak social interaction condition (27) is satisfied.

We shall now state conditions on the behaviour of individual agents and on the signal kernel $Q$ which guarantee asymptotic stability on the level of aggregate behaviour.

Theorem 4.7 Suppose that our Assumption 4.4 holds true, and that the weak social interaction condition (27) is satisfied. Then the macroscopic process $\left\{R\left(x_{t}\right)\right\}_{t \in \mathbb{N}}$ converges in law to a unique stationary distribution $\mu^{*}$. Here $\mu^{*}$ is a probability measures on $\mathcal{M}_{h}(E)$.

Proof: Due to Lemma 4.9 in Horst (2001a), our weak social interaction condition yields

$$
|u(\xi, s)-u(\hat{\xi}, s)| \leq \alpha d(\xi, \hat{\xi}) \quad\left(\xi, \hat{\xi} \in \mathcal{M}_{h}(E), s \in S\right) .
$$

Thus, the random system with complete connections $\Sigma^{*}$ introduced in (24) is distance diminishing in the sense of Definition 4.3 (ii). In view of (25), our assertion therefore follows from Theorem 4.1.2 in Norman (1972) as $\left(\mathcal{M}_{h}(E), d\right)$ is a compact metric space. 


\section{Dynamics of Equilibrium Prices}

We are now going to analyze the long run behaviour of stock prices in our financial market model. In order to simplify notation, and in view of (25), we shall from now on assume that the random environment $\{\tilde{\varrho}\}_{t \in \mathbb{N}}$ for the evolution of the asset price process $\left\{p_{t}\right\}_{t \in \mathbb{N}}$ is generated by the Markov chain $\left(\left\{\xi_{t}\right\}_{t \in \mathbb{N}},\left(\hat{\mathbb{P}}_{\xi}\right)_{\xi \in \mathcal{M}_{h}(E)}\right)$ associated with the random system with complete connections $\Sigma^{*}$ introduced in (24). More precisely, we consider a price process $\left\{p_{t}\right\}_{t \in \mathbb{N}}$ which is defined by the recursive relation (14), but assume that the conditional law

$$
\tilde{Q}\left(\xi_{t} ; \cdot\right)
$$

of the random variable $\tilde{\varrho}_{t}$ given the random field $\xi_{t}$ is described by a stochastic kernel $\tilde{Q}$ from $\mathcal{M}_{h}(E)$ to $\mathcal{M}(C)$. Throughout this section, we assume that the assumptions stated in Theorem 4.7 are satisfied.

In Section 5.1 we consider an ergodic reference model. We shall assume that the initial distribution of the Markov chain $\left\{\xi_{t}\right\}_{t \in \mathbb{N}}$ is given by its unique invariant distribution $\mu^{*}$. Under the law $\hat{\mathbb{P}}_{\mu^{*}}(\cdot):=\int \hat{\mathbb{P}}_{\xi}(\cdot) \mu^{*}(d \xi)$ the process $\left\{\xi_{t}\right\}_{t \in \mathbb{N}}$ is stationary and ergodic. In economic terms this amounts to a situation where the mood of the market is already in equilibrium. Imposing a suitable mean-contraction condition on the transformation $F$ we show that the sequence $\left\{p_{t}\right\}_{t \in \mathbb{N}}$ governed by (14) converges pathwise to a stationary process $\left\{P_{t}^{*}\right\}_{t \in \mathbb{N}}$ in the sense that

$$
\hat{\mathbb{P}}_{\mu^{*}}\left[\lim _{t \rightarrow \infty}\left|p_{t}-P_{t}^{*}\right|=0\right]=1 .
$$

Nonetheless, the price fluctuations in our financial market model may be highly volatile. Consider, for example the log-linear dynamics (12) and assume that $c_{c}>1$. In periods where the fraction of chartists who are involved in the formation of equilibrium prices comes close to the critical value $\frac{1}{c_{c}}$, the process $\left\{p_{t}\right\}_{t \in \mathbb{N}}$ may become highly unstable. Economically, this feature can be interpreted as the temporary occurrence of bubbles or crashes in a financial market model whose overall behaviour is ergodic.

In Section 5.2, we concentrate on a financial market model where the sequence of temporary price equilibria is governed by a log-linear stochastic difference equation of the form

$$
\log p_{t+1}=f\left(\tilde{\varrho}_{t+1}\right) \log p_{t}+g\left(\tilde{\varrho}_{t+1}\right) \quad(t \in \mathbb{N}) .
$$


We consider a situation where the mood of the market is out of equilibrium, but settles down in the long run. We state conditions on the non-stationary random environment $\left\{\tilde{\varrho}_{t}\right\}_{t \in \mathbb{N}}$ which ensure that the price process converges to a stationary regime.

\subsection{Equilibrium Prices in a Stationary Environment}

Let us analyze the long run behaviour of the asset price process in an ergodic reference model. We assume that the Markov chain $\left\{\xi_{t}\right\}_{t \in \mathbb{N}}$ which described the stochastic evolution of the aggregate behaviour already starts in its probabilistic equilibrium $\mu^{*}$; see Theorem 4.7. In this case, the sequence of temporary price equilibria $\left\{p_{t}\right\}_{t \in \mathbb{N}}$ is driven by a stationary and ergodic random environment $\left\{\tilde{\varrho}_{t}\right\}_{t \in \mathbb{N}}$, and so we can apply a result provided in Borovkov (1998) in order to study to asymptotics of the asset price process.

We denote by $p$ the initial price at time $t=0$, put $\tilde{\varrho}_{n}^{0}:=\left(\tilde{\varrho}_{1}, \ldots, \tilde{\varrho}_{n}\right)$ and assume that the following conditions concerning the iterates

$$
F\left(\tilde{\varrho}_{n}^{0}, p\right):=F\left(\tilde{\varrho}_{n}, \cdot\right) \circ \cdots \circ F\left(\tilde{\varrho}_{2}, \cdot\right) \circ F\left(\tilde{\varrho}_{1}, p\right) \quad(n \in \mathbb{N}) .
$$

are satisfied; see also Borovkov (1998), Chapter 2, Section 8.

Assumption $5.1 \quad$ (i) For some $p_{0} \in \mathbb{R}$ and for each $\delta>0$, there exists $N=N(\delta)$ such that, for all $n \geq 1$, we have

$$
\hat{\mathbb{P}}_{\mu^{*}}\left[\left|p_{0}-F\left(\tilde{\varrho}_{n}^{0}, p_{0}\right)\right|>N\right]<\delta .
$$

Thus, the sequence $\left\{p_{t}\right\}_{t \in \mathbb{N}}$ is assumed to be bounded in probability.

(ii) The function $F=F(\tilde{\varrho}, p)$ is continuous in $p$ and there exists an integer $r \geq 1$, a number $\beta>0$, and a measurable function $c: \mathbb{R}^{r} \rightarrow \mathbb{R}_{+}$such that

$$
\begin{aligned}
\left|F\left(\tilde{\varrho}_{r}^{0}, p_{1}\right)-F\left(\tilde{\varrho}_{r}^{0}, p_{2}\right)\right| & \leq c\left(\tilde{\varrho}_{r}^{0}\right)\left|p_{1}-p_{2}\right| \quad\left(p_{1}, p_{2} \in \mathbb{R}\right) \\
\frac{1}{r} \hat{\mathbb{E}}_{\mu^{*}} \log c\left(\tilde{\varrho}_{r}^{0}\right) & \leq-\beta
\end{aligned}
$$

where $\hat{\mathbb{E}}_{\mu^{*}}$ denotes the expectation with respect to the measure $\hat{\mathbb{P}}_{\mu^{*}}$.

(iii) The sequence $\left\{\log c\left(\tilde{\varrho}_{j r}, \ldots, \tilde{\varrho}_{j r+r}\right)\right\}_{j \in \mathbb{N}}$ satisfies the strong law of large numbers. 
Note that Assumption 5.1 (ii) may be viewed as a mean contraction condition for the sequence $\left\{p_{t}\right\}_{t \in \mathbb{N}}$ governed by (14). The next theorem follows from Theorem 12.2 in Borovkov (1998). In the case of a linear transformation $F$, it reduces to Theorem 1 in Brandt (1986).

Theorem 5.2 Suppose that Assumption 5.1 is satisfied. Then there exists a stationary process $\left\{P_{t}^{*}\right\}_{t \in \mathbb{N}}$ such that, for any starting point $p$ of the sequence $\left\{p_{t}\right\}_{t \in \mathbb{N}}$, we have

$$
\hat{\mathbb{P}}_{\mu^{*}}\left[\lim _{t \rightarrow \infty}\left|P_{t}^{*}-p_{t}\right|=0\right]=1 .
$$

If the initial value $p$ has the same distribution as the random variable $P_{0}^{*}$, then the price process $\left\{p_{t}\right\}_{t \in \mathbb{N}}$ is stationary.

For our financial market model, and under the assumption that the mood of the market is already in equilibrium, Theorem 5.2 provides a bound for the aggregate effect of interaction between different traders which ensures that the induced price fluctuations are asymptotically stationary. Beyond this bound the price process may become highly transient. A continuoustime analogue of Theorem 5.2 is formulated in Föllmer and Schweizer (1993), where it is shown that the trajectories in a simple log-linear model may either tend to zero or go off to infinity with positive probability if the destabilizing effects of the environment are on average too strong.

Example 5.3 Consider the log-linear demand structure described by (5) together with (6) and (7). In this case, the market clearing condition implies the log-linear price dynamics of the form

$$
\log p_{t+1}=f\left(\tilde{\varrho}_{t+1}\right) \log p_{t}+g\left(\tilde{\varrho}_{t+1}\right),
$$

where the mappings $f, g: \mathcal{M}(C) \rightarrow \mathbb{R}$ are given by (13). If the conditions

$$
\hat{\mathbb{E}}_{\mu^{*}} \log \left|f\left(\hat{\varrho}_{0}\right)\right|<0 \quad \text { and } \quad \hat{\mathbb{E}}_{\mu^{*}}\left(\log \left|g\left(\hat{\varrho}_{0}\right)\right|\right)^{+}<\infty
$$

are satisfied, then the price process converges pathwise to a stationary process. Such a log-linear price dynamics is analyzed in detail in Section 5.2.

Example 5.4 (Generalized auto-regression) Suppose that the sequence of equilibrium prices $\left\{p_{t}\right\}_{t \in \mathbb{N}}$ obeys the recurrence relation

$$
p_{t+1}=G\left(f\left(\tilde{\varrho}_{t+1}\right) \hat{G}\left(p_{t}\right)+g\left(\tilde{\varrho}_{t+1}\right)\right),
$$


where $\hat{G}, G: \mathbb{R} \rightarrow \mathbb{R}$ are Lipschitz continuous functions, i.e.,

$$
\left|\hat{G}\left(p_{1}\right)-\hat{G}\left(p_{2}\right)\right| \leq \hat{k}\left|p_{1}-p_{2}\right|, \quad\left|G\left(p_{1}\right)-G\left(p_{2}\right)\right| \leq k\left|p_{1}-p_{2}\right| .
$$

As a result of Theorems 8.4 and 12.2 in Borovkov (1998), the sequence $\left\{p_{t}\right\}_{t \in \mathbb{N}}$ satisfies parts (i) and (ii) of our Assumption 5.1 if

$$
\log |\hat{k} k|+\hat{\mathbb{E}}_{\mu^{*}} \log \left|f\left(\tilde{\varrho}_{0}\right)\right|<0 \quad \text { and } \quad \hat{\mathbb{E}}_{\mu^{*}}\left(\log \left|g\left(\tilde{\varrho}_{0}\right)\right|\right)^{+}<\infty .
$$

\subsection{Equilibrium Prices in a Non-Stationary Environment}

Let us now concentrate on a financial market model where both the individual excess demands functions and the reference levels take a log-linear form; see for instance (5) together with (6) and (7). In such a situation, the dynamics of our price fluctuations is described by a stochastic sequence $\left\{p_{t}\right\}_{t \in \mathbb{N}}$ which obeys the log-linear recursive relation

$$
\log p_{t+1}=f\left(\tilde{\varrho}_{t+1}\right) \log p_{t}+g\left(\tilde{\varrho}_{t+1}\right) \quad(t \in \mathbb{N}) .
$$

Our goal is to analyze the asymptotic behaviour of asset prices under the assumption that the mood of the market is out of equilibrium but becomes stationary in the long run.

In order to make this more precise, note first that the environment $\left\{\tilde{\varrho}_{t}\right\}_{t \in \mathbb{N}}$ for the evolution of the price process is stationary and ergodic under the law $\hat{\mathbb{P}}_{\mu^{*}}$. Next, we introduce the $\sigma$-fields

$$
\hat{\mathcal{F}}_{t}:=\sigma\left(\tilde{\varrho}_{s}: s \geq t\right)
$$

and denote by $\hat{\mathcal{T}}:=\bigcap_{t \in \mathbb{N}} \hat{\mathcal{F}}_{t}$ the tail-field generated by the sequence $\{\tilde{\varrho}\}_{t \in \mathbb{N}}$. We say that the process $\{\tilde{\varrho}\}_{t \in \mathbb{N}}$ has a nice asymptotic behaviour, if

$$
\lim _{t \rightarrow \infty} \sup _{\xi}\left\|\hat{\mathbb{P}}_{\xi}-\hat{\mathbb{P}}_{\mu^{*}}\right\|_{\hat{\mathcal{F}}_{t}}=0
$$

Here, $\left\|\hat{\mathbb{P}}_{\xi}-\hat{\mathbb{P}}_{\mu^{*}}\right\|_{\hat{\mathcal{F}}_{t}}$ denotes the total variation of the signed measure $\hat{\mathbb{P}}_{\xi}-\hat{\mathbb{P}}_{\mu^{*}}$ on $\hat{\mathcal{F}}_{t}$. Since the total variation distance is continuous along decreasing $\sigma$ algebras, we have

$$
\lim _{t \rightarrow \infty}\left\|\hat{\mathbb{P}}_{\xi}-\hat{\mathbb{P}}_{\mu^{*}}\right\|_{\hat{\mathcal{F}}_{t}}=\left\|\hat{\mathbb{P}}_{\xi}-\hat{\mathbb{P}}_{\mu^{*}}\right\|_{\hat{\mathcal{T}}}
$$


Thus, (31) implies that $\hat{\mathbb{P}}_{\xi}=\hat{\mathbb{P}}_{\mu^{*}}$ on $\hat{\mathcal{T}}$, and so the asymptotic behaviour of a nice driving sequence $\left\{\tilde{\varrho}_{t}\right\}_{t \in \mathbb{N}}$ is the same under $\hat{\mathbb{P}}_{\xi}$ and under $\hat{\mathbb{P}}_{\mu^{*}}$. In this sense the sequence $\{\tilde{\varrho}\}_{t \in \mathbb{N}}$ becomes stationary in the long run.

Lemma 5.6 below shows that the process $\left\{\tilde{\varrho}_{t}\right\}_{t \in \mathbb{N}}$ has a nice asymptotic behaviour whenever the Markov chain $\left\{\xi_{t}\right\}_{t \in \mathbb{N}}$ converges in law to a unique equilibrium and if the stochastic kernel $\tilde{Q}$ introduced in (28) satisfies a mild regularity condition. Using this result, we show in Theorem 5.7, that our financial price fluctuations behave asymptotically in a stable manner if the destabilizing effects of the random environment on the dynamics of the price process are on average not too strong.

Assumption 5.5 The stochastic kernel $\tilde{Q}$ from $\left(\mathcal{M}_{h}(E), d\right)$ to $\mathcal{M}(C)$ introduced in (28) satisfies a uniform Lipschitz condition:

$$
\|\tilde{Q}(\xi ; \cdot)-\tilde{Q}(\hat{\xi} ; \cdot)\| \leq L d(\xi, \hat{\xi}) .
$$

Let us now establish the following result about the asymptotic behaviour of the driving sequence $\left\{\tilde{\varrho}_{t}\right\}_{t \in \mathbb{N}}$.

Lemma 5.6 Suppose that our Assumptions 4.4 and 5.5 are satisfied. Then the random environment $\left\{\tilde{\varrho}_{t}\right\}_{t \in \mathbb{N}}$ for the evolution of the asset price process has a nice asymptotic behaviour in the sense of (31).

Proof: Let us denote by $\mathcal{B}$ the Borel- $\sigma$-field on $\left(\mathcal{M}_{h}(E), d\right)$. In a first step, we are going to establish the existence of a constant $L<\infty$ such that

$$
\left|\hat{\mathbb{P}}_{\xi}\left[\left\{\tilde{\varrho}_{0}, \ldots, \tilde{\varrho}_{t}\right\} \in B\right]-\hat{\mathbb{P}}_{\tilde{\xi}}\left[\left\{\tilde{\varrho}_{0}, \ldots, \tilde{\varrho}_{t}\right\} \in B\right]\right| \leq L d(\xi, \tilde{\xi})
$$

for all $t \in \mathbb{N}$ and $B \in \otimes_{i=1}^{t} \mathcal{B}$. To this end, we denote by $L_{Q}$ and $L_{\tilde{Q}}$ the Lipschitz constants for the stochastic kernels $Q$ and $\tilde{Q}$, respectively, and introduce the quantity

$$
\alpha_{t}:=\sup _{B} \sup _{\xi \neq \tilde{\xi}} \frac{\left|\hat{\mathbb{P}}_{\xi}\left[\left\{\tilde{\varrho}_{0}, \tilde{\varrho}_{2}, \ldots, \tilde{\varrho}_{t}\right\} \in B\right]-\hat{\mathbb{P}}_{\tilde{\xi}}\left[\left\{\tilde{\varrho}_{0}, \tilde{\varrho}_{2}, \ldots, \tilde{\varrho}_{t}\right\} \in B\right]\right|}{d(\xi, \tilde{\xi})} .
$$

Due to the contraction property of the transformation $u$ established in Theorem 4.7 and because of the uniform Lipschitz conditions imposed on the 
stochastic kernel $Q$ and $\tilde{Q}$, we have that

$$
\begin{aligned}
& \left|\hat{\mathbb{P}}_{\xi}\left[\left\{\tilde{\varrho}_{0}, \ldots, \tilde{\varrho}_{t+1}\right\} \in B\right]-\hat{\mathbb{P}}_{\tilde{\xi}}\left[\left\{\tilde{\varrho}_{0}, \ldots, \tilde{\varrho}_{t+1}\right\} \in B\right]\right| \\
\leq & \sup _{B}\left|\hat{\mathbb{P}}_{\xi}\left[\tilde{\varrho}_{0} \in B\right]-\hat{\mathbb{P}}_{\tilde{\xi}}\left[\tilde{\varrho}_{0} \in B\right]\right|+\sup _{B}|\hat{Q}(\xi ; B)-\hat{Q}(\tilde{\xi} ; B)| \\
& \sup _{B, s}\left|\hat{\mathbb{P}}_{u(\xi, s)}\left[\left\{\tilde{\varrho}_{0}, \ldots, \tilde{\varrho}_{t}\right\} \in B\right]-\hat{\mathbb{P}}_{u(\tilde{\xi}, s)}\left[\left\{\tilde{\varrho}_{0}, \ldots, \tilde{\varrho}_{t}\right\} \in B\right]\right| \\
\leq & \left(L_{\tilde{Q}}+L_{Q}+\alpha \alpha_{t}\right) d(\xi, \tilde{\xi}),
\end{aligned}
$$

and so

$$
\alpha_{t} \leq\left(L_{\tilde{Q}}+L_{Q}\right) \sum_{i \in \mathbb{N}} \alpha^{i} \leq \frac{L_{\tilde{Q}}+L_{Q}}{1-\alpha} .
$$

In particular, (32) holds with $L=\frac{L_{\tilde{Q}}+L_{Q}}{1-\alpha}$.

Let us denote by $U^{t}$ the t-fold iteration of the transition operator $U$ associated with the Markov chain $\left\{\xi_{t}\right\}_{t \in \mathbb{N}}$. Since

$$
\left\|\hat{\mathbb{P}}_{\xi}-\hat{\mathbb{P}}_{\mu^{*}}\right\|_{\hat{\mathcal{F}}_{t}}=\sup _{B}\left|\left(U^{t} \hat{\mathbb{P}}_{\bullet}\left[\left\{\tilde{\varrho}_{t}\right\}_{t \in \mathbb{N}} \in B\right]\right)(\xi)-\int \mathbb{P}_{\tilde{\xi}}\left[\left\{\tilde{\varrho}_{t}\right\}_{t \in \mathbb{N}} \in B\right] \mu^{*}(d \tilde{\xi})\right|
$$

and because the mapping $\xi \mapsto \mathbb{P}_{\xi}\left[\left\{\tilde{\varrho}_{t}\right\}_{t \in \mathbb{N}} \in B\right]$ from $\mathcal{M}_{h}(E)$ to $[0,1]$ is Lipschitz continuous with Lipschitz constant $L<\infty$, we can apply Lemma 2.1.57 in Iosefescu and Theodorescu (1968): There are constants $\bar{L}<\infty$ and $\bar{\alpha}<1$ such that

$$
\sup _{\xi, B}\left|\left(U^{t} \hat{\mathbb{P}}_{\bullet}\left[\left\{\tilde{\varrho}_{t}\right\}_{t \in \mathbb{N}} \in B\right]\right)(\xi)-\int_{\mathcal{M}_{h}(E)} \mathbb{P}_{\tilde{\xi}}\left[\left\{\tilde{\varrho}_{t}\right\}_{t \in \mathbb{N}} \in B\right] \mu^{*}(d \tilde{\xi})\right| \leq \bar{L} \bar{\alpha}^{t} .
$$

This yields our assertion.

In our present setting, the logarithmic stock price process is described by a linear recursive stochastic equation in a non-stationary environment. Under the assumption that the environment has a nice asymptotic behaviour, the asymptotics of such processes is analyzed in Horst (2001b). These results allow us to introduce a bound for the aggregate effect of interaction between different traders which ensures that the price process is driven into equilibrium whenever the mood of the market itself settles down in the long. 
Theorem 5.7 Suppose that the asset price process $\left\{p_{t}\right\}_{t \in \mathbb{N}}$ obeys the loglinear relation (30) and that our Assumptions 4.4 and 5.5 are satisfied. If the random variables $f\left(\tilde{\varrho}_{0}\right)$ and $g\left(\tilde{\varrho}_{0}\right)$ satisfy

$$
\hat{E}_{\mu^{*}} \log \left|f\left(\tilde{\varrho}_{0}\right)\right|<0 \quad \text { and } \quad \hat{E}_{\mu^{*}}\left(\log \left|g\left(\tilde{\varrho}_{0}\right)\right|\right)^{+}<\infty
$$

then there exists a unique probability measure $\nu$ on $\mathbb{R}^{\mathbb{N}}$ such that the shifted sequence $\left\{p_{t+T}\right\}_{t \in \mathbb{N}}$ converges in distribution to $\nu$ as $T \rightarrow \infty$.

Proof: Due to (31) and (33), the sequence $\left\{\left(f\left(\tilde{\varrho}_{t}\right), g\left(\tilde{\varrho}_{t}\right)\right)\right\}_{t \in \mathbb{N}}$ is "nice" in the sense of Definition 2.1 in Horst (2001b). Thus, our assertion follows from Theorem 2.4 in Horst (2001b).

\section{Continuous-Time Asset Price Processes}

In this section, we shall again assume that the dynamics of the logarithmic price process can be described by linear recursive stochastic equation. Under mild technical assumptions on the driving sequence $\left\{\tilde{\varrho}_{t}\right\}_{t \in \mathbb{N}}$ we will obtain a continuous-time asset price process $\left\{P_{t}\right\}_{t \geq 0}$ by passage to the limit from the discrete-time equilibrium price process $\left\{p_{t}\right\}_{t \in \mathbb{N}}$ defined recursively by

$$
\log p_{t+1}-\log p_{t}=f\left(\tilde{\varrho}_{t+1}\right) \log p_{t}+g\left(\tilde{\varrho}_{t+1}\right) \quad(t \in \mathbb{N}) .
$$

The convergence concept we use is weak convergence on the Skorohood space $\mathbb{D}^{d}$ of all $\mathbb{R}^{d}$-valued right-continuous functions with left limits on $[0, \infty)$, endowed with the weak topology. A similar approach was carried out by Föllmer and Schweizer (1993) who passed to a continuous-time model from a sequence of suitably specified discrete-time processes evolving in an $e x-$ ogenously given stationary and ergodic random environment. We extend the Föllmer-Schweizer model by (i) analyzing a situation were the driving sequence is derived endogenously, and (ii) by replacing the stationarity assumption on the mood of the market by an asymptotic stability condition.

To this end, we consider in Section 6.1 a sequence of discrete-time stochastic processes $\left\{P^{n}\right\}_{n \in \mathbb{N}}, P^{n}=\left\{P_{t}^{n}\right\}_{t \in \mathbb{N}}$, defined recursively by the linear relation

$$
P_{t+1}^{n}-P_{t}^{n}=\frac{1}{\sqrt{n}} A_{t} P_{t}^{n}+\frac{1}{\sqrt{n}} B_{t} \quad(t, n \in \mathbb{N})
$$


in the random environment $\left\{\left(A_{t}, B_{t}\right)\right\}_{t \in \mathbb{N}}$. We formulate conditions on the non-stationary driving sequence $\left\{\left(A_{t}, B_{t}\right)\right\}_{t \in \mathbb{N}}$ which allow us to derive a convergence result for the processes $\left\{P^{n}\right\}_{n \in \mathbb{N}}$. This will be achieved by applying an invariance principle to the non-stationary continuous-time processes $X^{n}$ and $Y^{n}$ which are specified by

$$
X_{t}^{n}:=\frac{1}{\sqrt{n}} \sum_{i=0}^{[n t]} A_{i} \quad \text { and } \quad Y_{t}^{n}:=\frac{1}{\sqrt{n}} \sum_{i=0}^{[n t]} B_{i} .
$$

Armed with these results, we establish in Section 6.2 a Black-Scholes type approximation for the asset price process (34) in a situation where the mood of the market settles down in the long run.

In the sequel it will be convenient to denote by $\operatorname{Law}(X, \mathbb{P})$ the law of a random variable $X$ under the measure $\mathbb{P}$.

\subsection{A Functional Central Limit Theorem for Non-Stationary Sequences}

Let $\left\{\left(A_{t}, B_{t}\right)\right\}_{t \in \mathbb{N}}$ a sequence of $\mathbb{R}^{2}$-valued random variables defined on some probability space $(\Omega, \mathcal{F}, \mathbb{P})$. For any $n \in \mathbb{N}$, we consider a discrete-time process $\left\{P_{t}^{n}\right\}_{t \in \mathbb{N}}$ given by the linear relation

$$
P_{t+1}^{n}-P_{t}^{n}=\frac{1}{\sqrt{n}} A_{t} P_{t}^{n}+\frac{1}{\sqrt{n}} B_{t} .
$$

If $\left\{Z_{t}^{n}\right\}_{t \in \mathbb{N}}$ is any discrete-time process, we identify $Z^{n}$ with the continuoustime process $Z_{t}^{n}:=Z_{[n t]}^{n}(t \geq 0)$ whose paths are right-continuous. In terms of the quantities $X^{n}$ and $Y^{n}$ defined in (35), our stochastic difference equation (36) is equivalent to the stochastic differential equation

$$
d P_{t}^{n}=P_{t-}^{n} d X_{t}^{n}+d Y_{t}^{n}
$$

If the driving sequence $\left\{\left(A_{t}, B_{t}\right)\right\}_{t \in \mathbb{N}}$ is stationary and ergodic under the law $\mathbb{P}$, and under what Föllmer and Schweizer (1993) call "standard assumptions" on the two sources of randomness $\left\{A_{t}\right\}_{t \in \mathbb{N}}$ and $\left\{B_{t}\right\}_{t \in \mathbb{N}}$, one can apply an invariance principle to the sequences $X^{n}$ and $Y^{n}(n \in \mathbb{N})$ defined by (35) and assume that the process $\left\{\left(X^{n}, Y^{n}\right)\right\}_{n \in \mathbb{N}}$ is "good" in the sense of the following definition. 
Definition 6.1 (Duffie and Protter (1992)) A sequence $\left\{Z^{n}\right\}_{n \in \mathbb{N}}$ of semimartingales defined on probability spaces $\left(\Omega^{n}, \mathcal{F}^{n}, \mathbb{P}^{n}\right)$ is called "good" if, for any sequence $\left\{H^{n}\right\}_{n \in \mathbb{N}}$ of càdlàg adapted processes, the convergence

$$
\operatorname{Law}\left(\left(Z^{n}, H^{n}\right), \mathbb{P}^{n}\right) \stackrel{w}{\longrightarrow} \operatorname{Law}((Z, H), \mathbb{P}) \quad(n \rightarrow \infty)
$$

implies the convergence

$$
\operatorname{Law}\left(\left(Z^{n}, H^{n}, \int H_{-}^{n} d Z^{n}\right), \mathbb{P}^{n}\right) \stackrel{w}{\rightarrow} \operatorname{Law}\left(\left(Z, H, \int H_{-} d Z\right), \mathbb{P}\right) .
$$

Here $\stackrel{w}{\longrightarrow}$ denotes weak convergence of probability measures.

Let us summarizes some results from Föllmer and Schweizer (1993).

Proposition 6.2 $\quad$ (i) Suppose that the driving sequence $\left\{\left(A_{t}, B_{t}\right)\right\}_{t \in \mathbb{N}}$ is stationary and ergodic and that $\mathbb{E} A_{0}=\mathbb{E} B_{0}=0$. Under "standard assumptions" on the two sources of randomness $\left\{A_{t}\right\}_{t \in \mathbb{N}}$ and $\left\{B_{t}\right\}_{t \in \mathbb{N}}$, the sequence $\left\{\left(X^{n}, Y^{n}\right)\right\}_{n \in \mathbb{N}}$ introduced in (35) converges in law to the Gaussian martingale $(X, Y)=V \cdot W$. Here, $W=\left(W_{1}, W_{2}\right)$ denotes a two-dimensional standard Brownian motion and $V$ is a suitable deterministic $2 \times 2$ dispersion matrix.

(ii) If the sequence $\left\{\left(X^{n}, Y^{n}\right)\right\}_{n \in \mathbb{N}}$ is "good" in the sense of Definition 6.1, the process $\left\{\left(X^{n}, Y^{n}, P^{n}\right)\right\}_{n \in \mathbb{N}}$ converges in law to $(X, Y, P)$. Here, $P=\left\{P_{t}\right\}_{t \geq 0}$ is the unique strong solution of the stochastic differential equation

$$
d P_{t}=P_{t} d X_{t}+d Y_{t}
$$

That is, $P=\left\{P_{t}\right\}_{t \geq 0}$ is the pathwise solution of a SDE of the form

$$
d P_{t}=\sigma P_{t} d W_{t}+\tilde{\sigma} d \tilde{W}_{t}
$$

where $W, \tilde{W}$ are standard Brownian motions with correlation $\varrho$. If $\sigma>$ 0 , then the diffusion limit $\left\{P_{t}\right\}_{t \geq 0}$ converges to a stationary process, and its invariant distribution can be given in closed form.

We are now going to establish a "non-stationary" version of Proposition 6.2. We obtain a convergence result for the processes $\left\{P^{n}\right\}_{n \in \mathbb{N}}$ given that the driving sequence $\left\{\left(A_{t}, B_{t}\right)\right\}_{t \in \mathbb{N}}$ is out of equilibrium, but has a nice asymptotic behaviour. 
Assumption 6.3 $\quad$ (i) There exists a probability measure $\mathbb{P}^{*}$ on $(\Omega, \mathcal{F})$ such that the environment $\left\{\left(A_{t}, B_{t}\right)\right\}_{t \in \mathbb{N}}$ is stationary and ergodic under the law $\mathbb{P}^{*}$.

(ii) The asymptotic behaviour of the environment $\left\{\left(A_{t}, B_{t}\right)\right\}_{t \in \mathbb{N}}$ is the same under $\mathbb{P}$ and under $\mathbb{P}^{*}$, i.e.,

$$
\left\|\mathbb{P}-\mathbb{P}^{*}\right\|_{\mathcal{T}}=\lim _{t \rightarrow \infty}\left\|\mathbb{P}-\mathbb{P}^{*}\right\|_{\hat{\mathcal{F}}_{t}}=0
$$

where $\hat{\mathcal{F}}_{t}:=\sigma\left(\left(A_{s}, B_{s}\right): s \geq t\right)$ and $\mathcal{T}:=\bigcap_{t \in \mathbb{N}} \hat{\mathcal{F}}_{t}$ is the tail-field generated by the sequence $\left\{\left(A_{t}, B_{t}\right)\right\}_{t \in \mathbb{N}}$.

(iii) We have $\mathbb{E}^{*} A_{0}=\mathbb{E}^{*} B_{0}=0$, where $\mathbb{E}^{*}$ denotes the expectation with respect to the law $\mathbb{P}^{*}$.

(iv) Under the law $\mathbb{P}^{*}$ an invariance principle can be applied to the sequence $\left\{\left(X^{n}, Y^{n}\right)\right\}_{t \in \mathbb{N}}$ given by (35).

Below, we will show that the driving sequence $\left\{\left(f\left(\tilde{\varrho}_{t}\right), g\left(\tilde{\varrho}_{t}\right)\right)\right\}_{t \in \mathbb{N}}$ for the asset price process defined by (34) satisfies parts (i), (ii) and (iv) of the above assumption whenever our Assumptions 4.4 and 5.5 are satisfied. In this case the driving sequence also satisfies one of the "standard assumptions". This allows us to obtain a diffusion approximation for the asset price process (34).

Theorem 6.4 If the driving sequence $\left\{\left(A_{t}, B_{t}\right)\right\}_{t \in \mathbb{N}}$ satisfies Assumption 6.3 , then the sequence of processes $\left\{Z^{n}\right\}_{n \in \mathbb{N}}=\left\{\left(X^{n}, Y^{n}\right)\right\}_{n \in \mathbb{N}}$ converges in distribution to the Gaussian martingale $(X, Y)=V \cdot W$. Here, $W=$ $\left(W_{1}, W_{2}\right)$ is a 2-dimensional standard Brownian motion under the law $\mathbb{P}^{*}$ and $V$ is a deterministic volatility matrix.

Proof: In order to verify our assertion, we proceed in several steps.

1. Due to our Assumption 6.3, we know that

$$
\operatorname{Law}\left(Z^{n}, \mathbb{P}^{*}\right) \stackrel{w}{\rightarrow} \operatorname{Law}\left(V \cdot W, \mathbb{P}^{*}\right) \quad(n \rightarrow \infty) .
$$

2. We shall now use the assumption that the asymptotic behaviour of the driving sequence $\left\{\left(A_{t}, B_{t}\right)\right\}_{t \in \mathbb{N}}$ is the same under $\mathbb{P}^{*}$ and under the original measure $\mathbb{P}$ in order to show that the sequences $\left\{X^{n}\right\}_{n \in \mathbb{N}}$ 
and $\left\{Y^{n}\right\}_{n \in \mathbb{N}}$ satisfy an invariance principle $\mathbb{P}$. More precisely, we are going to verify that

$$
\operatorname{Law}\left(Z^{n}, \mathbb{P}\right) \stackrel{w}{\longrightarrow} \operatorname{Law}\left(V \cdot W, \mathbb{P}^{*}\right) \quad(n \rightarrow \infty) .
$$

To this end, let $\left\{\sigma_{n}\right\}_{n \in \mathbb{N}}$ be a sequence of real numbers such that $\sigma_{n} \uparrow \infty$ and such that $\sigma_{n} / \sqrt{n} \rightarrow 0$ as $n \rightarrow \infty$. For a given "time horizon" $T>0$, and for each $n \in \mathbb{N}$, we introduce the two-dimensional process $\left\{\widetilde{Z}_{t}^{n}\right\}_{0 \leq t \leq T}$ given by

$$
\widetilde{Z}_{t}^{n}:= \begin{cases}\frac{1}{\sqrt{n}} \sum_{i=\sigma_{n}}^{[n t]}\left(A_{i}, B_{i}\right) & \text { if } \frac{\sigma_{n}}{\sqrt{n}} \leq t \leq T \\ 0 & \text { otherwise. }\end{cases}
$$

We denote by $d_{0}(\cdot, \cdot)$ and $\mathcal{B}_{\mathbb{D}}$ the Skorohood metric ${ }^{5}$ and the Borel- $\sigma$ field on the space $\mathbb{D}_{\mathbb{R}^{2}}[0, T]$, respectively. Note that

$$
d_{0}\left(Z^{n}, \widetilde{Z}^{n}\right) \leq \frac{\sigma_{n}}{\sqrt{n}}\left|\left(\frac{1}{\sigma_{n}} \sum_{i=0}^{\sigma_{n}}\left|A_{i}\right|, \frac{1}{\sigma_{n}} \sum_{i=0}^{\sigma_{n}}\left|B_{i}\right|\right)\right| .
$$

Since $\mathbb{P}=\mathbb{P}^{*}$ on the tail-field generated by the sequence $\left\{\left(A_{t}, B_{t}\right)\right\}_{t \in \mathbb{N}}$ and because the environment $\left\{\left(A_{t}, B_{t}\right)\right\}_{t \in \mathbb{N}}$ is ergodic under the law $\mathbb{P}^{*}$, the series

$$
\frac{1}{\sigma_{n}} \sum_{i=0}^{\sigma_{n}}\left|A_{i}\right| \text { and } \frac{1}{\sigma_{n}} \sum_{i=0}^{\sigma_{n}}\left|B_{i}\right|
$$

are $\mathbb{P}$ - and $\mathbb{P}^{*}$-almost surely convergent as $n \rightarrow \infty$. Since $\lim _{n \rightarrow \infty} \frac{\sigma_{n}}{\sqrt{n}}=$ 0 we obtain that

$$
\lim _{n \rightarrow \infty} d_{0}\left(Z^{n}, \widetilde{Z}^{n}\right)=0 \quad \mathbb{P} \text {-a.s. and } \mathbb{P}^{*} \text {-a.s. }
$$

Observe now that the event $\left\{\widetilde{Z}^{n} \in B\right\}\left(B \in \mathcal{B}_{\mathbb{D}}\right)$ belongs to the $\sigma$ algebra $\hat{\mathcal{F}}_{\sigma_{n}}$. Since the environment has a nice asymptotic behaviour in the sense of (31), there exists a sequence $\left\{c_{n}\right\}_{n \in \mathbb{N}}, c_{n} \downarrow 0$ as $n \rightarrow \infty$ such that

$$
\sup _{B}\left|\mathbb{P}\left[\widetilde{Z}^{n} \in B\right]-\mathbb{P}^{*}\left[\widetilde{Z}^{n} \in B\right]\right| \leq c_{n}
$$

\footnotetext{
${ }^{5}$ For the definition of $d_{0}$ see, e.g., Billingsley (1968), p. 113.
} 
3. Let us now denote by $Q^{*}$ the law of the Gaussian martingale $V \cdot W$ under the measure $\mathbb{P}^{*}$ and fix a $Q^{*}$-continuous set $B \in \mathcal{B}_{\mathbb{D}}$. By Step 1 above we know that

$$
\lim _{n \rightarrow \infty} \mathbb{P}^{*}\left[Z^{n} \in B\right]=Q^{*}[B] .
$$

Thus, due to (41) and due to Theorem 4.2 in Billingsley (1968), we have that

$$
\lim _{n \rightarrow \infty} \mathbb{P}^{*}\left[\widetilde{Z}^{n} \in B\right]=Q^{*}[B] .
$$

Using (42) we see that

$$
\lim _{n \rightarrow \infty} \mathbb{P}\left[\widetilde{Z}^{n} \in B\right]=Q^{*}[B] .
$$

Therefore, (41) and Theorem 4.2 in Billingsley (1968) imply that

$$
\operatorname{Law}\left(Z^{n}, \mathbb{P}\right) \stackrel{w}{\rightarrow} \operatorname{Law}\left(V \cdot W, \mathbb{P}^{*}\right) \quad(n \rightarrow \infty) .
$$

Remark 6.5 It is straightforward to extend the above theorem to the case $\mathbb{E}^{*} A_{0} \neq 0, \mathbb{E}^{*} B_{0} \neq 0$. For notational convenience, however, we shall restrict our attention to the case $\mathbb{E}^{*} A_{0}=\mathbb{E}^{*} B_{0}=0$.

Let us assume that the non-stationary driving sequence $\left\{\left(A_{t}, B_{t}\right)\right\}_{t \in \mathbb{N}}$ defined on $(\Omega, \mathcal{F}, \mathbb{P})$ is "good" in the sense of Duffie and Protter (1992) and satisfies our Assumption 6.3. In this case it follows from Theorem 6.4 and Proposition 6.2 that the sequence of processes $\left\{P^{n}\right\}_{n \in \mathbb{N}}$ defined by (37) converges in distribution to the unique strong solution of the stochastic differential equation

$$
d P_{t}=P_{t} d X_{t}+d Y_{t}
$$

Armed with these results we are now ready to show how the discrete-time asset price process $\left\{p_{t}\right\}_{t \in \mathbb{N}}$ in a financial market model with log-linear excess demand functions can indeed be approximated in law by a diffusion process. 


\subsection{A Diffusion Approximation for the Stock Price Process}

Let us return to the stock price process described by (34). Throughout this section we assume that Markov chain $\left\{\xi_{t}\right\}_{t \in \mathbb{N}}$ associated with the random system with complete connections $\Sigma^{*}$ defined by (24) converges in law to $\mu^{*}$ and that the random environment $\left\{\tilde{\varrho}_{t}\right\}_{t \in \mathbb{N}}$ for the evolution of the stock price process has a nice asymptotic behaviour in the sense of (31). This condition is satisfied if, for example, our Assumptions 4.4 and 5.5 hold true. Uniqueness of the stationary probability measure implies that the sequence

$$
\psi:=\left\{\left(f\left(\tilde{\varrho}_{t}\right), g\left(\tilde{\varrho}_{t}\right)\right)\right\}_{t \in \mathbb{N}}
$$

is stationary and ergodic under the law $\mathbb{P}_{\mu^{*}}$, and so the environment $\psi$ satisfies Assumption 6.3 (i) and (ii).

It follows from the proof of Lemma 5.6 that $\psi$ is $\varphi$-mixing under $\hat{\mathbb{P}}_{\xi}$ and that, independent of $\xi \in \mathcal{M}_{h}(E)$, the $n$-th mixing coefficient is bounded above by $L \alpha^{n}$. Hence, due to (2.58) and (2.59) in Billingsley (1968), the driving sequence $\psi$ also satisfies part (iv) of Assumption 6.3 whenever the random variables $f\left(\tilde{\varrho}_{0}\right)$ and $g\left(\tilde{\varrho}_{0}\right)$ have finite variance under $\hat{\mathbb{P}}_{\mu^{*}}$. Thus, we have shown

Theorem 6.6 Suppose that the random variables $f\left(\tilde{\varrho}_{0}\right)$ and $g\left(\tilde{\varrho}_{0}\right)$ have $f$ nite variance under $\hat{\mathbb{P}}_{\mu^{*}}$ and that $\hat{\mathbb{E}}_{\mu^{*}} f\left(\tilde{\varrho}_{0}\right)=\hat{\mathbb{E}}_{\mu^{*}} f\left(\tilde{\varrho}_{0}\right)=0$. Then the sequence $\left\{\left(X^{n}, Y^{n}\right)\right\}_{n \in \mathbb{N}}$ defined on the probability space $\left(\hat{\Omega}, \hat{\mathcal{F}}, \hat{\mathbb{P}}_{\xi}\right)$ by

$$
X_{t}^{n}:=\frac{1}{\sqrt{n}} \sum_{i=0}^{[n t]} f\left(\tilde{\varrho}_{i}\right) \quad \text { and } \quad Y_{t}^{n}:=\frac{1}{\sqrt{n}} \sum_{i=0}^{[n t]} g\left(\tilde{\varrho}_{i}\right) \quad(t \geq 0)
$$

converges in law to the continuous martingale $(X, Y)=V \cdot W$. Here $W=$ $\left(W_{1}, W_{2}\right)$ is a two-dimensional Brownian motion under $\hat{\mathbb{P}}_{\mu^{*}}$, and $V$ is a deterministic $2 \times 2$ dispersion matrix.

In order to obtain a diffusion approximation for our asset price process $\left\{p_{t}\right\}_{t \in \mathbb{N}}$ defined by (36), it remains to verify that the sequence $\left\{\left(X^{n}, Y^{n}\right)\right\}_{n \in \mathbb{N}}$ defined in (44) is indeed "good" in the sense of Duffie and Protter (1992).

Proposition 6.7 Under Assumptions 4.4 and 5.5 the sequence $\left\{Z^{n}\right\}_{n \in \mathbb{N}}=$ $\left\{\left(X^{n}, Y^{n}\right)\right\}_{n \in \mathbb{N}}$ of semimartingales given by (44) is "good" in the sense of Duffie and Protter (1992). 
Proof: In order to show that the sequence $\left\{Z^{n}\right\}_{n \in \mathbb{N}}$ is "good", we have to find a suitable semimartingale decomposition of the processes $Z^{n}(n \in \mathbb{N})$ under the law $\hat{\mathbb{P}}_{\xi}$. In the sequel, $\hat{\mathbb{E}}_{\xi}$ denotes the expectation with respect to $\hat{\mathbb{P}}_{\xi}$. Following Ethier and Kurtz (1986), and Duffie and Protter (1992), we introduce the $\sigma$-fields

$$
\mathcal{G}_{t}:=\sigma\left(\left\{f\left(\tilde{\varrho}_{i}\right), g\left(\tilde{\varrho}_{i}\right)\right\}: 0 \leq i \leq t\right) \quad(t \in \mathbb{N})
$$

and the processes $M=\left\{M_{t}\right\}_{t \in \mathbb{N}}$ and $A=\left\{A_{t}\right\}_{t \in \mathbb{N}}$ given by

$$
M_{t}:=\left(\begin{array}{c}
\sum_{k=0}^{t} f\left(\tilde{\varrho}_{k}\right)+\sum_{k=0}^{\infty} \hat{\mathbb{E}}_{\xi}\left[f\left(\tilde{\varrho}_{k+t}\right) \mid \mathcal{G}_{t}\right] \\
\sum_{k=0}^{t} g\left(\tilde{\varrho}_{k}\right)+\sum_{k=0}^{\infty} \hat{\mathbb{E}}_{\xi}\left[g\left(\tilde{\varrho}_{k+t}\right) \mid \mathcal{G}_{t}\right]
\end{array}\right)
$$

and

$$
A_{t}:=\left(\begin{array}{c}
\sum_{k=0}^{\infty} \hat{\mathbb{E}}_{\xi}\left[f\left(\tilde{\varrho}_{k+t}\right) \mid \mathcal{G}_{t}\right] \\
\sum_{k=0}^{\infty} \hat{\mathbb{E}}_{\xi}\left[g\left(\tilde{\varrho}_{k+t}\right) \mid \mathcal{G}_{t}\right]
\end{array}\right)
$$

Since the sequence $\left\{\tilde{\varrho}_{t}\right\}_{t \in \mathbb{N}}$ is $\varphi$-mixing under the law $\hat{\mathbb{P}}_{\xi}$, and because the n-th mixing coefficient is bounded above by $L \alpha^{n}$, the series in (45) and (46) are almost surely absolutely convergent; see, e.g., Ethier and Kurtz (1986), p. 351. Furthermore, $M$ is a vector of square integrable martingales with respect to the measure $\hat{\mathbb{P}}_{\xi}$ and the filtration $\left\{\mathcal{G}_{t}\right\}_{t \in \mathbb{N}}$. In terms of the quantities

$$
M^{n}=\left\{M_{[n t]}\right\}_{t \geq 0}, \quad \text { and } \quad A^{n}=\left\{A_{[n t]}\right\}_{t \geq 0}
$$

we consider the following decomposition:

$$
Z_{t}^{n}=\frac{1}{\sqrt{n}} M_{t}^{n}-\frac{1}{\sqrt{n}} A_{t}^{n} \quad(t \geq 0) .
$$

According to Theorem 4.3 in Duffie and Protter (1992), the sequence of semimartingales $\left\{Z^{n}\right\}_{n \in \mathbb{N}}$ is "good" as soon as the following condition is satisfied ("Condition B"):

$$
\sup _{n \in \mathbb{N}}\left\{\hat{\mathbb{E}}_{\xi}\left[\sup _{t \leq T}\left|\Delta M_{t}^{n}\right|\right]\right\}<\infty \quad \text { and } \quad \sup _{n \in \mathbb{N}}\left\{\hat{\mathbb{E}}_{\xi}\left[\left|A^{n}\right|_{T}\right]\right\}<\infty .
$$

Here, $\left|A_{T}^{n}\right|$ denotes the total variation of the process $A^{n}$ on the time interval $[0, T]$ and $\Delta M_{t}^{n}:=M_{t}^{n}-M_{t-}^{n}$. Obviously, the martingale $M^{n}$ has uniformly 
bounded expected jumps. Using standard estimates provided in Duffie and Protter (1992), Example 6.3, it follows that

$$
\sup _{n \in \mathbb{N}}\left\{\mathbb{E}_{\xi}\left[\left|A^{n}\right|_{T}\right]\right\}<\infty
$$

Thus, "Condition B" is satisfied, and so the sequence $\left\{Z^{n}\right\}_{n \in \mathbb{N}}$ is indeed "good".

Combining Theorem 6.6 with Proposition 6.7 leads us to the final result of this paper:

Theorem 6.8 Suppose that the random variables $f\left(\tilde{\varrho}_{0}\right)$ and $g\left(\tilde{\varrho}_{0}\right)$ have $f$ nite variance under $\hat{\mathbb{P}}_{\mu^{*}}$, that our Assumptions 4.4 and 5.5 are satisfied and that

$$
\hat{E}_{\mu^{*}} f\left(\tilde{\varrho}_{0}\right)=\hat{E}_{\mu^{*}} g\left(\tilde{\varrho}_{0}\right)=0 .
$$

Then the asset price process $\left\{p_{t}\right\}_{t \in \mathbb{N}}$ defined by (34) can be approximated in law by the continuous-time diffusion process $\left\{P_{t}\right\}_{t \geq 0}$ which solves the stochastic differential equation

$$
d P_{t}=P_{t} d X_{t}+d Y_{t}
$$

Here, $(X, Y)=V \cdot W$, where $W=\left(W_{1}, W_{2}\right)$ is a two-dimensional standard Brownian motion under the law $\hat{\mathbb{P}}_{\mu^{*}}$, and $V$ is a deterministic dispersion matrix. The diffusion limit $\left\{P_{t}\right\}_{t \geq 0}$ converges to a stationary regime and its invariant distribution can be given in closed from.

\section{Conclusion}

We have provided a unified probabilistic framework which allows us to analyse the long run behaviour of stock prices in a situation where the demand for a risky asset results from the interaction of a large number boundedly rational of agents. In our model, agents interact locally with their neighbors, and, at the same give, receive random signals about the average behaviour throughout the entire population. Under simplifying assumptions on their excess demand functions, and given that the interaction between different agents is not too strong, we have shown that the asset price process (i) converges to a unique equilibrium distribution and (ii) can be approximated in 
law by a diffusion process. In this sense the present paper provides another microeconomic foundation for the use of diffusion processes in the analysis of financial price fluctuations.

In our model, all randomness was generated endogenously, but can easily be extended to situations where the behaviour of agents also depends on exogenous economic fundamentals like interest rates or government spending. In a simple mean field type model, i.e., under the assumption that $N(a)=\{a\}$, such a situation is analyzed in Horst (1999). Numerical simulations show that slight changes in economic fundamentals may lead to large and sudden price fluctuations even though the price process becomes stationary in the long run. These fluctuations do not reflect rational adjustments to new information, but are due to a distinct herd behaviour.

\section{References}

BACHELIER, L. (1900): "Théorie de la Spéculation," Annales Scientifiques de l'École Normale Supérieur, 17, 21-86.

Barnsley, M. F., S. G. Demko, J. H. Elton, and J. S. Geronimo (1988): "Invariant Measures for Markov Processes Arising from Iterated Function Systems with Place-Dependent Probabilities," Ann. Inst. Henri Poincare, 24, 367-394.

Billingsley, P. (1968): Convergence of Probability Measures. John Wiley \& Sons, New York.

Borovkov, A. A. (1998): Ergodicity and stability of stochastic processes. John Wiley \& Sons, New York.

BRANDT, A. (1986): "The Stochastic Equation $Y_{n+1}=A_{n} Y_{n}+B_{n}$ with Stationary Coefficients," Adv. Appl. Probab., 18, 211-220.

Brock, W. A., And C. Hommes (1997): "A Rational Route to Randomness," Econometrica, 65 (5), 1059-1095.

Duffie, D., and P. Protter (1992): "From Discrete- to Continuous-Time Finance," Mathematical Finance, 2, 1-15. 
EthieR, S. N., And T. G. KuRTz (1986): Markov Processes Characterisation and Convergence. John Wiley \& Sons, New York.

FöllmeR, H. (1974): "Random Economies with many interacting agents," Journal of Mathematical Economics, 1, 51-62.

(1994): "Stock price fluctuation as a diffusion in a random environment," Philos. Trans. R. Soc. Lond., Ser. A, 1684, 471-483.

Föllmer, H., AND U. Horst (2001): "Convergence of Locally and Globally Interacting Markov Chains," Discussion paper 21, SFB 373, Humboldt Universität zu Berlin (http://sfb.wiwi.hu-berlin.de/papers/); submitted for publication.

Föllmer, H., ANd M. SchweIzer (1993): "A Microeconomic Approach to Diffusion Models for Stock Prices," Mathematical Finance, 3, 1-23.

Horst, U. (1999): "Ergodic Fluctuations in a Financial Market Model with Interacting Agents - The Mean Field Case," Discussion paper 106, SFB 373, Humboldt-Universität zu Berlin (http://sfb.wiwi.huberlin.de/papers/).

Horst, U. (2000): "Asymptotics of Locally and Globally Interacting Markov Chains Arising in Microstructure Models of Financial Markets," Ph.D. thesis, Humboldt-Universiät zu Berlin.

Horst, U. (2001a): "Asymptotics of Locally Interacting Markov Chains with Global Signals," Discussion paper 29, SFB 373, Humboldt Universität zu Berlin (http://sfb.wiwi.hu-berlin.de/papers/); submitted for publication.

(2001b): "The Stochastic Equation $Y_{t+1}=A_{t} Y_{t}+B_{t}$ with NonStationary Coefficients," J. Appl. Prob., 38, 80-95.

Iosefescu, M., And M. Theodorescu (1968): Random Processes and Learning. Springer-Verlag, Berlin.

Kirman, A. (1998): "On the Transitory Nature of Gurus," Working paper, EHESS et Université de Marseille III. 
Kreps, D. (1982): Multiperiod Securities and the efficient allocation of Risk: A Comment on the Black-Scholes Option Procing Model. In: The economics of uncertainty and information (ed. J.McCall), University of Chicago Press, Chicago.

Lasota, A., and J. A. York (1994): "Lower Bound Technique for Markov Operators and iterated Function Systems," Random Comput. Dyn., 2 (1), $41-77$.

Lux, T. (1998): "The Socio-Economic Dynamics of Speculative Markets: Interacting Agents, Chaos, and the Fat Tails of Return Distributions," Journal of Economic Behavior and Organization, 33, 143-165.

Lux, T., AND M. MARChesi (1999): "Scaling and Criticality in a Stochastic Multi-Agent Model of a Financial Market," Nature, 370, 498-500.

Norman, F. M. (1972): Markov Processes and Learning Models. Academic Press, New York.

Vasserstein, L. N. (1969): "Markov Processes over Denumerable Product of Spaces Describing large Systems of Automata," Problemy Peredaci Informacii, 5, 64-72. 